\title{
GENERAL THEORY FOR FLOW OPTIMISATION OF SPLIT- FLOW THIN FRACTIONATION
}

\author{
Yonghao Zhang \\ Centre for Microfluidics, CLRC Daresbury Laboratory, Warrington WA4 4AD, UK \\ FAX: 0044-1925-603634 \\ Email: Y.Zhang@dl.ac.uk
}




\title{
GENERAL THEORY FOR FLOW OPTIMISATION OF SPLIT-FLOW THIN FRACTIONATION
}

\author{
Yonghao Zhang and David R. Emerson \\ Centre for Microfluidics, CLRC Daresbury Laboratory, Warrington WA4 4AD, UK \\ Jason M Reese \\ Department of Mechanical Engineering, University of Strathclyde, Glasgow G1 1TX, UK
}

\begin{abstract}
Recently, magnetic split-flow thin (SPLITT) fractionation has been developed to separate macromolecules, colloids, cells and particles. However, the previous theory, developed for an infinitely long channel, needs to be improved to consider the flow transit regimes at both inlet and outlet. In this paper, we describe a new approach to optimising flow rates for particle separation which considers the effect of flow transit region. Surprisingly, the critical particle migration velocities derived by the present theory are identical to the previous simplified theory. Therefore, the previous simplified theory may have wider application than might have been expected. As a test of our theory, a numerical simulation based on solving Navier-Stokes equations has also been carried out for a magnetic SPLITT device. The trajectory of a particle with the critical migration velocity is exactly as expected by our theory. Following experimental validation, this work will facilitate the design of new SPLITT fractionation systems with smaller aspect ratio.
\end{abstract}

Keywords: Split-flow thin fractionation; Computational fluid dynamics

\section{Introduction}

Split-flow thin (SPLITT) fractionation is a technique whereby an external field is used to separate macromolecules, colloids, cells and particles in sample solutions. It is widely used in biological, 
medical, environmental and industrial applications [1-7]. A schematic of a typical SPLITT fractionation system is shown in figure 1 (where the magnetic field is shown). Generally, the system has two inlets and two outlets with controlled but different flow rates. Two inflows emerge from the inlet splitter, and the flow is split again at the outlet splitter. A sample solution enters through the inlet $a$ with a carrier fluid injected at the inlet $b$. Particles with higher mobility will be separated and collected at the outlet $b$ due to an applied field, e.g. a magnetic field. The driving force for this particle migration could also be due to gravity or electrical fields etc.

Currently, SPLITT fractionation systems are designed to have a very large ratio of channel length to thickness, i.e. $L / W>>1$. The theory of this fractionation was developed by assuming the streamlines through the system are parallel, and the velocity profile at the separation channel is parabolic as in a fully-developed state (the flow transit regimes at the channel entrance and outlet are ignored). The position of two imaginary inlet and outlet splitting streamlines, i.e. ISS and OSS in figure 1, are determined by solving the fully-developed velocity profile equation [8]. In this paper, a twodimensional description is adopted because of the large channel width, $b$, compared to the channel thickness, $W$. The resulting theory has been used to optimise flow rates, and the results are usually in good agreement with experimental data $[3,4,7]$.

Fuh et al. [9] have investigated the effect of the shape of the splitter at the inlet to the flow field at different Reynolds numbers. With increasing Reynolds number, they found that the flow development length (or the flow transit length) at the inlet increases, and vortices can be generated if the Reynolds number increases further, causing flow mixing. Therefore, the fully-developed flow assumption of the previous theory is not sufficient in the case of a small $L / W$ channel or a large Reynolds number, and the velocity field in the transit regime cannot be described by a parabolic profile [9]. As a first order estimate, the length of the flow transit region at the inlet, $l_{d}$, can be given 
by $(0.625+0.044 \mathrm{Re}) W$. Further details can be found in reference [10]. Moreover, as we show below, any particle will undergo a rapid change in the transit regime which is contradictory to the assumption of the previous theory where the particles are assumed to follow the streamlines until the flow develops fully.

Since, e.g., the magnetic field intensity can be carefully designed, the field-induced particle migration velocity can also be controlled. Therefore, the particle migration time from up-stream of ISS to sub-stream of OSS could be shorter if a larger migration velocity can be generated. This leads to the prospect of more rapid separation by reducing the length of channel. The advantages of having a shorter SPLITT fractionation channel are dramatic reduction of operation time and possible miniaturisation of the device. In such a case, because a larger field force can be used, the influence of the hydrodynamic lift force on the particle separation performance may become insignificant. Moreover, a smaller $L / W$ may allow more flexibility in device fabrication. Because the Reynolds number increases with channel thickness, the velocity of the carrier fluid is constrained in order to ensure smooth streamlines. This may cause the particles to take a significantly long time to pass through the channel, especially in industrially scaled-up devices. So, reducing the channel length may be one of the few choices available to reduce particle pass-through time. Increasing the channel thickness $W$ may also help to align the channel physical layers more precisely, so that optimised device operation conditions can be achieved.

If we reduce the length of the SPLITT fractionation channel, the effect of the flow transit regime becomes more significant. Fuh et al. [9] have shown that the shape and size of the flow splitters play a negligible role if the flow is strictly laminar — which is true for most practical flow rates. Therefore, we can treat the splitters as thin walls in the laminar flow regime. Fuh et al. [9] also showed that the streamlines are not parallel in the transit regimes near the two splitters, and the 
fluid is not just moving in the $y$ direction (see figure 1), i.e. the fluid velocity in the $x$ direction is not zero as assumed in the previous theory. As a result, the field-induced particle migration velocity cannot be assumed to be perpendicular to the fluid velocity. Therefore, there is now a need to develop a general theory to accommodate these flow transit regimes.

\section{General theory of SPLITT fractionations}

The fluid flow, ignoring the effect of particles, can be described by the Navier-Stokes equation. However, there is no analytical solution available for the flow at the entry and outlet regimes. From the numerical simulation results given by Fuh et al. [9], it could be a substantial distance along the channel before the flow is fully-developed, i.e. a parabolic velocity profile and streamlines parallel to each other. In the theory developed below, we relax the restriction of $L / W>>1$ which was assumed in the previous theory $[4,5,7]$.

As in previous work, we take the Reynolds number based on the channel thickness, $W$, to be small, so that the flow is laminar. The particle concentration should also be small $(<0.1 \%$ of volume $)$ in order to limit the effect of particles on the fluid flow [11]. The particle size is large enough that Brownian motion can be neglected and small enough that the relaxation time of the particle to the flow field is very small, compared to the characteristic time scale of the flow field. Therefore, the particle diameter needs to be greater than $1 \mu \mathrm{m}[12]$ and have a Stokes number far less than unity [13]. The Stokes number, St, is defined as the ratio of particle relaxation time to the characteristic time of the flow field, i.e.

$$
S t=\frac{\tau_{r}}{\tau_{f}}<<1
$$

where $\tau_{r}$ is the particle relaxation time for a Stokes flow, given by 
$\tau_{r}=\frac{\rho_{p} d^{2}}{18 \mu}$

where $d$ and $\rho_{p}$ are the diameter and density of the particle, respectively, $\mu$ is the fluid viscosity, and $\tau_{f}$ is the characteristic time of the flow field. For channel flow, $\tau_{f}$ is estimated via

$$
\tau_{f}=\frac{W}{\langle V\rangle},
$$

where $\langle V\rangle$ is the mean fluid velocity in the channel.

The lift force, especially near the channel wall, has been proven to have a significant effect on the particle trajectory [14-18]. The Saffman lift force due to the velocity gradient can be estimated by [18]:

$f_{\text {Saff }}=1.61 \mu d|U-V| \sqrt{\operatorname{Re}_{f}}$,

where $U$ is the particle velocity, and

$\operatorname{Re}_{f}=\frac{\rho_{f} d^{2}}{\mu}|\nabla V|$,

where $\rho_{f}$ is the fluid density. The particle Reynolds number, $R e_{p}$, is defined as $\frac{\rho_{f} d}{\mu}|U-V|$. For $\operatorname{Re}_{p}<<\sqrt{\operatorname{Re}_{f}}<1.0$, the ratio of the Saffman lift force to the drag force can be estimated as [19]

$\frac{f_{\text {Saff }}}{f_{\text {drag }}}=\frac{K}{12 \pi} \sqrt{\operatorname{Re}_{f}}$,

where $K$ is a constant with value 6.46. In a channel with cross-sectional averaged fluid velocity of $2.0 \mathrm{~mm} / \mathrm{s}$, for a $10 \mu \mathrm{m}$ diameter particle moving in a water-based liquid, the Saffman lift force is typically less than $1 \%$ of the drag force. Only for large particles with high shear rate would this extra lift become substantial. Consequently, we will ignore the lift force here. 
The particle diameter is assumed to be much smaller than the channel thickness, therefore we treat a particle as a point. First we introduce a streamline function, $\psi(x, y)$, for steady flow which is defined via

$$
V_{x}=\frac{\partial \psi}{\partial y} \text { and } V_{y}=-\frac{\partial \psi}{\partial x}
$$

where $V_{x}$ and $V_{y}$ are the field velocities in the $x$ and $y$ direction respectively, and $\psi(x, y)=\operatorname{constant}$ is a streamline. The volume flow rate per unit width, $Q_{1-2}$, through any two points $\left(x_{1}, y_{1}\right)$ and $\left(x_{2}, y_{2}\right)$ in the flow field can be described by

$Q_{1-2}=\psi\left(x_{2}, y_{2}\right)-\psi\left(x_{1}, y_{1}\right)$

The velocity of the carrier fluid depends on the position $(x, y)$. The two imaginary streamlines ISS and OSS may not be parallel to the $y$ direction, so that the distance of ISS or OSS to the wall varies with $y$. Generally, the particle velocity at any point has the two components

$U_{y}(x, y)=V_{y}(x, y) \quad$ and $\quad U_{x}(x, y)=V_{x}(x, y)+U_{c}$,

where $U_{c}$ is the field induced-velocity of the particle relative to the carrier fluid. Here we assume the induced particle migration velocity is in the $x$ direction only. In a short time, $d t$, the particle travels distance $d x$ in the $x$ direction:

$d x=\left[V_{x}(x, y)+U_{c}\right] d t$.

In this same period of time, the particle travels by $d y$ in the $y$ direction:

$d y=V_{y}(x, y) d t$

From equations (10) and (11), we obtain

$V_{x}(x, y) d y-V_{y}(x, y) d x=-U_{c} d y$.

Introducing equation (7), we get

$$
\frac{\partial \psi}{\partial y} d y+\frac{\partial \psi}{\partial x} d x=-U_{c} d y .
$$


If the particle moves out of the channel without touching the wall, then we integrate equation (13) from $y=L$ to 0 to obtain

$\psi\left(x_{1}, L\right)-\psi\left(x_{2}, 0\right)=-U_{c} L$,

where the field induced particle migration velocity $U_{c}$ is treated as a constant (the integration result will depend on the particle trajectory if $U_{c}$ is space-dependent). If the particle touches the wall before it reaches the end of the channel, then the integration becomes,

$\psi\left(x_{1}, L\right)-\psi\left(W / 2, y_{2}\right)=-U_{c} L$

The critical field-induced particle velocity, $U_{c 1}$, for a particle trajectory that starts from the bottom of the splitter at the inlet $a$ and ends at the splitter at the outlet $b$ is

$U_{c 1}=\frac{Q_{o u t}^{a}-Q_{\text {in }}^{a}}{b L}$

If the particles have a migration velocity smaller than $U_{c 1}$, they will all move out through the outlet $a$. In the case of separating fragile cells or if we want to reduce the number of particles sticking to the wall and increase the particle retrieval rate (if a magnetic field is used), another critical particle migration velocity is important, $U_{c 2}$, viz.

$U_{c 2}=\frac{Q_{i n}^{b}}{b L}$

Under this critical migration velocity, a particle starting from the inlet splitter can just reach the outlet wall, so that any particle with smaller migration velocity from the inlet $a$ will not hit the outlet wall. The critical velocity, $U_{c 3}$, for a particle starting at the wall of the inlet $a$ and ending at the splitter of the outlet $b$ is

$$
U_{c 3}=\frac{Q_{o u t}^{a}}{b L} .
$$

Particles with migration velocity larger than $U_{c 3}$ will be collected at the outlet $b$. These three critical migration velocities are essential for optimisation of operation flow rates. 
The present theory removes the assumption of negligibly small flow transit regimes at the splitters, providing the flow remains laminar, i.e. flow laminas will not be mixed up. Surprisingly, the critical particle migration velocities given by equations (16-18) are exactly the same as the previous simplified theory. This may be the reason that the previous theory has been in very good agreement with the measurement. Therefore, the previous simplified theory has wider application and can also be applied to shorter aspect ratio devices without introducing unnecessary error due to the effect of the transit region. The significance of this approach is that it overcomes the theoretical hurdle for exploiting short $L / W$ SPLITT devices. The present approach also helps us to understand the relation between the separation rate and the flow lamina for mono-dispersed particles. The space monodistribution of particles before the entrance is no longer valid in the fully-developed regime as assumed in the previous theory [4].

\section{An example of scale-up of a magnetic SPLITT device}

A magnetic particle sorter has been developed by Fuh and Chen $[3,20]$ and a similar annular channel magnetic SPLITT fractionation device has also been developed [21]. These devices have been used to separate magnetic beads-labelled cells etc. Due to the possibility of increasing the magnetic field-induced particle migration velocity, reducing the length of device is feasible. For constant particle migration velocity, the present general theory agrees well with the previous theory for large aspect ratio devices, and much experimental and numerical validation for the previous theory can be found in the literature $[4,6,22]$. Here, we propose a magnetic SPLITT, which is similar to the device designed by Fuh and his coworkers [3, 20, 23], but with short aspect ratio. Optimisation of operation conditions will be thoroughly discussed by applying the present theory. Numerical experiments which solve the Navier-Stokes equations will be carried out and compared with the present theory. The advantages of numerical experiment are that we can investigate a wide 
range of design parameters e.g. flow rates, channel dimensions, geometries, fluid properties etc. Therefore, numerical experiments can identify the major design issues and optimise the design, while significantly reducing the experimental cost and compressing the development cycle. In addition, they can reveal particle trajectories which are difficult to measure and have not been rigorously investigated.

\section{Magnetic field and particle migration velocity}

In order to negate the effect of the gravity, we assume the device will be operated in a parallel mode, as suggested by Fuh and Chen [3]. The force acting on the particle in the $x$ direction can be described by

$m_{p} \frac{d V_{x}}{d t}=F_{d r a g}+F_{m}$

where $m_{p}$ is the mass of the particle, $F_{d r a g}$ and $F_{m}$ are the drag force and the Kelvin force (due to an external magnetic field) respectively. Inter-particle hydrodynamic interaction is neglected as we assume a low particle concentration. For a Stokes flow, the drag force is $3 \pi \mu d U_{c}$. For a particle with magnetic permeability $\mu_{m}$ freely suspended in an external magnetic field $H$, the Kelvin force is given by [24]

$F_{m}=\frac{1}{2} \nabla\left(\mu_{m} H^{2}\right)$

The external magnetic field $H$ is related to the magnetic flux density $B$ as $H=B / \mu_{m}$, where the magnetic flux density $B$ can be determined by [24]

$\nabla \cdot B=0$.

Therefore, we can determine the Kelvin force if we know the particle magnetic permeability $\mu_{m}$ and the external magnetic field, $H$. 
Here, we introduce "magnetophoretic mobility", a term proposed in the literature $[21,25,26]$. The particle migration velocity in an aqueous media under a magnetic field can be given by

$U_{c}=m S_{m}$,

where $m$ is the particle magnetophoretic mobility, and the local force field strength $S_{m}$ is given by

$$
S_{m}=\left|\nabla\left(\frac{B^{2}}{2 \mu_{0}}\right)\right|,
$$

where $\mu_{0}$ is the magnetic permeability of the vacuum. The particle magnetophoretic mobility can be measured by a device called a CTV [21]. Fuh et al. used a pair of rare-earth magnets (Nd-Fe-B) to generate a uniform local force field [23].

\section{Numerical simulation and discussion}

The Navier-Stokes equations which govern the motion of the fluid phase can be given by [27]:

Continuity equation: $\quad \frac{\partial \rho}{\partial t}+\nabla \cdot(\rho \boldsymbol{V})=0$

Momentum equation: $\rho \frac{D \boldsymbol{V}}{D t}=-\nabla p+\mu \nabla^{2} \boldsymbol{V}+\rho \boldsymbol{g}$,

where $\boldsymbol{g}$ is the gravity. Since one-way coupling is assumed, i.e. particles have negligible effect on the flow field, the Navier-Stokes equations are solved for the steady flow in the channel. The particle trajectory is determined by solving equations (9) and (22), because the particles respond instantaneously to the change of fluid motion. In this paper, results from a code written in Fortran 90, using the classical SIMPLE algorithm, for solving Navier-Stokes equations are presented. Details of this simulation technique can be found widely, e.g. Ferziger and Perić [28].

Numerical experiment can clearly show the particle trajectories, which are difficult to measure experimentally. Moreover, experimental measurement may not rule out the influence of other factors such as device alignment precision etc. The Navier-Stokes equations are capable of 
capturing the laminar flow with low Reynolds number, so that numerical examination is one method for evaluating the present theory. The following numerical simulation is of a separation channel with length, $L=20 \mathrm{~mm}$, thickness $W=2 \mathrm{~mm}$, and width $b=20 \mathrm{~mm}$. The length of both inlet and outlet splitter is $5 \mathrm{~mm}$. Because inlet and outlet channels are $1 \mathrm{~mm}$ thick, the flow can developed fully before it enters the main separation channel.

In the separation process, volume flow rates are optimised as control parameters. Fixed volume flow rates are therefore set as boundary conditions at both inlets and outlets. The velocity profile is assumed uniform at the inlets $a, b$, and fully-developed at the outlets $a, b$. At the splitters and the walls, non-slip boundary conditions are adopted, and the thickness of the splitters is assumed to be very small.

Suppose we have two types of magnetic beads-labelled cells with magnetophoretic mobilities, $m_{1}$, $m_{2}$, of $1.0 \times 10^{-3}$ and $3.0 \times 10^{-3} \mathrm{~mm}^{3} /$ TAs respectively, and the magnetic field we apply gives an $S_{m}$ of $3 \times 10^{8} \mathrm{TA} / \mathrm{m}^{2}$. In order to optimise flow rates to ensure separation, we need to evaluate the critical magnetophoretic mobilities, $m_{c 1}, m_{c 2}, m_{c 3}$, corresponding to critical induced migration velocities $U_{c 1}, U_{c 2}, U_{c 3}$. The following criteria need to be satisfied: $m_{1}<m_{c 1}$, so that cells with magnetophoretic mobility of $m_{1}$ will be collected at the outlet $a ; m_{c 3}<m_{2}<m_{c 2}$, so that cells with magnetophoretic mobility of $m_{2}$ will move out of the outlet $b$ without sticking to the wall. An operating condition with $Q_{\text {in }}^{a}=5.0 \mathrm{ml} / \mathrm{min}, Q_{\text {in }}^{b}=25.0 \mathrm{ml} / \mathrm{min}, Q_{\text {out }}^{a}=15.0 \mathrm{ml} / \mathrm{min}$ and $Q_{\text {out }}^{b}=15.0$ $\mathrm{ml} / \mathrm{min}$ is imposed so the critical magnetophoretic mobilities, $m_{c 1}, m_{c 2}$ and $m_{c 3}$ are therefore $1.39 \times 10^{-3}, 3.47 \times 10^{-3}$ and $2.08 \times 10^{-3} \mathrm{~mm}^{3} / \mathrm{TAs}$ respectively.

Figure 2 shows the calculated ISS and OSS streamlines, and three representative particle trajectories which have the critical magnetophoretic mobilities $m_{c 1}=1.39 \times 10^{-3}, m_{c 2}=3.47 \times 10^{-3}$ and 
$m_{c 3}=2.08 \times 10^{-3} \mathrm{~mm}^{3} /$ TAs respectively. In figures $3-5$, the particle trajectories are only shown in the main separation channel, i.e. $0 \leq y / W \leq 10$. However, the boundary conditions for the inlets and outlets are set at $y / W$ of 12.5 and -2.5 respectively. We see the transit regime at the separation channel inlet is a significant proportion of the whole flow field. Moreover, the particles have already deviated largely from the corresponding streamlines with the same initial starting point at the inlet splitter when the flow develops fully. For example, if we compare the ISS streamline with trajectory $m_{c 1}$, or with $m_{1}$ and $m_{2}$ in figure 3, we find the particle trajectories have departed from ISS significantly in the transit regime. Therefore, the numerical analysis can clearly distinguish the insufficient assumption that was made in the previous theory, even though it is difficult to test experimentally. These particle trajectories are consistent with the present theory predictions, e.g. the particle with $m_{c 1}$ starts from the inlet splitter and ends exactly at the outlet splitter as expected.

When $m_{1}<m_{c 1}$ and $m_{c 3}<m_{2}<m_{c 2}$ the particles will be separated, according to the present theory. ISS and OSS streamlines and the particle trajectories with the magnetophoretic mobilities $m_{1}, m_{2}$ are shown in figure 3 and confirm our predictions. We can see that all the cells with $m_{2}$, starting from any position between the inlet wall and the splitter, will all move into the outlet $b$ within the two limits of the trajectories. Even when the initial position is at the inlet splitter, the particle with $m_{1}$ cannot pass the OSS streamline and moves into the outlet $b$, so that all the particles with $m_{1}$ will be collected at the outlet $a$ regardless of their initial positions at the inlet $a$. Moreover, the particles will not hit the wall, which would cause a reduction in the cell retrieval rate.

For the same device, if we set up arbitrary operation conditions as shown in figure 4, we can work out the critical magnetophoretic mobilities and determine the range of separable particles. Typical operating flow rates of $Q_{i n}^{a} / Q_{\text {in }}^{b}=1 / 5, Q_{\text {out }}^{b} / Q_{\text {out }}^{a}=1 / 5$ are chosen and the calculated critical magnetophoretic mobilities $m_{c 1}, m_{c 2}$ and $m_{c 3}$ are $5.56 \times 10^{-4}, 6.94 \times 10^{-4}$ and $6.94 \times 10^{-4} \mathrm{~mm}^{3} / \mathrm{TAs}$ 
respectively. Particles with magnetophoretic mobility less than $5.56 \times 10^{-4} \mathrm{~mm}^{3} / \mathrm{TAs}$ can be clearly separated from the ones larger than $6.94 \times 10^{-4} \mathrm{~mm}^{3} /$ TAs. However, because $m_{c 2}$ and $m_{c 3}$ are the same, some particles with larger magnetophoretic mobility will reach and stick to the wall. As a result, these operation flow rates will reduce cell retrieval rate and are not optimised.

\section{Conclusions}

A theory with a more general approach for flow rate optimisation of SPLITT thin fractionation devices has been developed. It relaxes the previous assumption of an infinitely large aspect ratio of the channel length to the channel thickness. Numerical experiments have shown that the particle trajectories are consistent with the predictions of the present theory, so that it can be used to guide new design and optimise the operation conditions. This theory awaits comprehensive experimental validation, but until then it is a useful guide to the design of more efficient SPLITT fractionation systems.

\section{Acknowledgements}

The authors are grateful to Dr R. Johnstone for his valuable technical suggestion. This work was financially supported by the Medical Research Council of UK under grant reference 57719. 


\section{References}

[1] J.V. Giddings, Science 260 (1993) 1456.

[2] C.B. Fuh, Anal. Chem. 72 (2000) 266A.

[3] C.B. Fuh, S.Y. Chen, J. Chromatogr. A 857 (1999) 193.

[4] S.R. Springston, M.N. Myers, J.C. Giddings, Anal. Chem. 59 (1987) 344.

[5] P.S. Williams, Separation Sci. Tech. 29 (1994) 11.

[6] P. Cardot, S. Batta, A. Sinon, C. Delage, J. Chromatogr. B. 768 (2002) 285.

[7] C. Cantado, P. Reschiglian, S. Faccini, A. Zattoni, F. Dondi, J. Chromatogr. A 871 (2000) 449.

[8] P.S. Williams, S. Levin, T. Lenczycki, J.C. Giddings, Ind. Eng. Chem. Res. 31 (1992) 2172.

[9] C.B. Fuh, E.M. Trujillo, J.C. Giddings, Separation Sci. Tech. 30 (1995) 3861.

[10] B. Atkinson, M.P. Brocklebank, C.C.H. Card, J.M. Smith, AIChE J. 15 (1969) 548.

[11] S.E. Elghobashi, Appl. Sci. Research 52 (1994) 309.

[12] R.J. Hunter — Foundations of Colloid Science, Second Edition, Oxford Press, New York, 2001.

[13] C. Crowe, M. Sommerfield, Y. Tsuji - Multiphase flows with droplets and particles, CRC Press, London, 1998.

[14] P.S. Williams, M.H. Moon, J.C. Giddings, Colloids and Surfaces A: Physicochemical and Eng. Aspects 113 (1996) 215.

[15] P.S. Williams, Y.H. Xu, P. Reschiglian, J.C. Giddings, Anal. Chem. 67 (1997) 349.

[16] P.S. Williams, T. Koch, J.C. Giddings, Chem. Eng. Communications 111 (1992) 121.

[17] J. Zhang, P.S. Williams, M.N. Myers, J.C. Giddings, Sep. Sci. Tech. 29 (1994) 2493.

[18] P.G. Saffman, J. Fluid Mech. 31 (1968) 624.

[19] L.S. Fan, C. Zhu — Principles of gas-solid flows, Cambridge University Press, Cambridge, 1998.

[20] C.B. Fuh, S. Chen, J. Chromatogr. A 813 (1998) 313.

[21] M. Hoyos, L.R. Moore, K.E. McCloskey, S. Margel, M. Zuberi, J.J. Chalmers, M. Zborowski, J. Chromatogr. A 903 (2000) 99.

[22] P.S. Williams, Separation Sci. Tech. 29 (1994) 11.

[23] C.B. Fuh, J.Z. Lai, C.M. Chang, J. Chromatogr. A 923 (2001) 263. 
[24] H.E. Knoepfel - Magnetic fields: a comprehensive theoretical treatise for practical use, John Willey \& Sons Inc., New York, 1999.

[25] P.S. Williams, M. Zborowski, J.J. Chalmers, Anal. Chem. 71 (1999) 3799.

[26] M. Zborowski, L. Sun, L.R. Moore, P.S. Williams, J.J. Chalmers, J. Magnetism Magn. Mater. 194 (1999) 224.

[27] G.K. Batchelor — An introduction to fluid dynamics, Cambridge University Press, Cambridge, 1967.

[28] J.H. Ferziger, M. Perić — Computational methods for fluid dynamics ( $3^{\text {rd }}$ edition), Springer, Berlin, 2002.

\section{Figure legends}

Figure 1. Schematic of a magnetic SPLITT Fractionation device

Figure 2. Particle trajectories and ISS, OSS streamlines in the separation channel, where $m_{c 1}=1.39 \times 10^{-3}, m_{c 2}=3.47 \times 10^{-3}$ and $m_{c 3}=2.08 \times 10^{-3} \mathrm{~mm}^{3} /$ TAs respectively, and $Q_{i n}^{a}=5.0, Q_{i n}^{b}=25.0$, $Q_{\text {out }}^{a}=15.0$ and $Q_{\text {out }}^{b}=15.0 \mathrm{ml} / \mathrm{min}$ respectively.

Figure 3. Particle trajectories and ISS, OSS streamlines in the separation channel, where $m_{1}=1.0 \times 10^{-3} \mathrm{~mm}^{3} /$ TAs and $m_{2}=3.0 \times 10^{-3} \mathrm{~mm}^{3} / \mathrm{TAs}$, and $Q_{\text {in }}^{a}=5.0, Q_{\text {in }}^{b}=25.0, Q_{\text {out }}^{a}=15.0$ and $Q_{\text {out }}^{b}$ $=15.0 \mathrm{ml} / \mathrm{min}$ respectively.

Figure 4. Particle trajectories and ISS, OSS streamlines in the separation channel, where $m_{c 1}=5.56 \times 10^{-4}, m_{c 2}=6.94 \times 10^{-4}$ and $m_{c 3}=6.94 \times 10^{-4} \mathrm{~mm}^{3} /$ TAs respectively, and $Q_{i n}^{a}=1.0, Q_{i n}^{b}=5.0$, $Q_{\text {out }}^{a}=5.0$ and $Q_{\text {out }}^{b}=1.0 \mathrm{ml} / \mathrm{min}$ respectively. 